\title{
Animal research facility for conducting GLP study
}

\begin{abstract}
The wide range of chemicals which are in everyday use - such as medicines or household products - as well as chemicals used in manufacturing, or fertilisers and pesticides used in farming, must be tested to make sure that they are as safe as possible for animals and people. Safety testing makes up a very small proportion of animal research and is done to guard against products which could cause damage like cancer or birth defects. This is essential for the health of people, animals and the environment. As well as protecting the consumer, workers involved in production and those that come into contact with chemicals during transport must be protected. Diseases must be diagnosed as accurately and quickly as possible so that the most effective treatment can be given. Animal experiments have been vital in developing techniques such as scanning to check on the health of unborn babies, to identify some cancers, to diagnose heart disease and in research leading to many important blood tests. Good Laboratory Practice (GLP) is essential to carry out preclinical/non clinical studies where in studies are planned, performed, reported, monitored, and archived for its quality and integrity. Therefore it is mandatory for any organisation to have Animal Research Facility as per GLP norms. Facility should be suitable and convenient to conduct toxicology-preclinical animal study. In addition to animal facility there should be laboratories required for supporting animal facility activities.
\end{abstract}

Volume 4 Issue $3-2018$

\author{
Pradeep Deshmukh \\ PRADO-Preclinical Research and Development \\ OrganisationPune, India
}

\section{Correspondence: Pradeep Deshmukh, PRADO-Preclinical Research and Development organisation pune, India,} Email pradeepdeshmukh1949@gmail.com

Received: May 22, 2018| Published: May 29, 2018

Keywords: good laboratory practice, animal research facility, planned, performed, reported, monitored

\section{Introduction}

Good Laboratory Practice (GLP) started when the FDA issued mandatory GLP requirements in June 1979. A little later, the OECD introduced the OECD Principles for GLP concerning the safety testing of any chemical substance. Any company either in US or Europe wishing to register medicines had to perform safety studies in compliance with US FDA or OECD. GLP is a regulation covering the quality management of non-clinical safety studies. The aim of the regulation is to encourage scientists to organise and perform their studies in a way which promotes the quality and validity of the test data. There is a difference between the "science" of a study and the "organization" of a study. GLP requires that the scientists responsible for the organization of studies implement clear structures, responsibilities and procedures in compliance with GLP so that the test data are more reliable. GLP also promotes international recognition of study data. When studies are performed according to OECD GLP Principles, 30 countries of the world (OECD member states), who have accepted the GLP Principles, must recognize that the data from these studies have been generated under acceptable organizational standards. Even non-OECD member states are willing to accept the reliability of data resulting from GLP compliant studies. Mutual recognition of results refers to the fact that GLP is an internationally accepted set of regulations for the conduct of studies. GLP requires that test facilities be of appropriate size, construction and location to meet the requirements of the study and minimize disturbances that would interfere with the validity of the study. They should be designed to provide an adequate degree of separation between the various activities of the study.

\section{Animal research facility}

The facility should be designed and operated to control selected parameters (such as temperature, humidity and light) to minimize the effects of environmental variables on the animal. In addition, the facility should be organised in a way that prevents the animals from coming into contact with disease, or with a test item other than the one under investigation. Animal research facility should have separations maintained by species, studies, quarantine change rooms, receipt of test materials and its storage, feed and bedding, cage washing area, necropsy and waste disposal. The environment control system should maintain the temperature, humidity and airflow constantly at the defined levels for the species concerned. Entry to animal research facility should be restricted to only the staff dedicated to work in the animal rooms. The staff should use dedicated personnel protective like coat, gloves, face mask, cap etc. Outside foot wares should not be allowed inside the animal research facility. There should be clean and dirty corridors.

\section{Three "R" principles}

Research on laboratory animals is necessary for scientific research for generating new knowledge and information. There is considerable amount of evidence showing how animal research benefited to human and animals. However over a period of time scientists realised use of animals should be justified. Hence, the concept of Three "R" was evolved by Russell and Burch (1959) which explains Replacement, Reduction and Refinement. 
Replacement: methods which avoid or replace the use of animals in Research.

Reduction: use of methods that enable researchers to obtain comparable levels of information from fewer animals, or to obtain more information from the same number of animals.

Refinement: use of methods that alleviate or minimize potential pain, suffering or distress, and enhance animal welfare for the animals used.

An ethically responsible consideration must be made in the face of the conflict of interests between man and animal. Before scientists begin their activities, all research in which laboratory animals are used must be presented to the Institutional Animal Ethics Committee for ethical assessment. Laboratory animal testing is not permitted without IAEC approval. Animal housing systems designed and managed to protect the animals from infections with unwanted agents coming from outside the barrier. The widespread use of the term barrier in laboratory animal science started in the early 1960 s, primarily in reference to rodent-breeding facilities and programs designed to produce "specific pathogen-free" rats and mice for research. As the confounding impact of infection with a growing number of agents on research outcomes became increasingly apparent, barrier housing for rodents has become standard for housing research animals in addition to breeding colonies.

\section{Biomedical research}

In the last 30 years, the development and use of transgenic and gene-targeted (knockout) mouse models rapidly increased the demand for rodent barrier housing space in biomedical research facilities to protect these unique animals. Most new animal facilities for biomedical research have a significant rodent barrier component, and it is common for new facilities to be entirely dedicated to housing rodents under barrier conditions. Barriers may consist of single or double corridor layers of protection, with both physical and management components. Use of multiple layers reduces risks; however, where people are involved, there is no such thing as a totally protected, risk-free facility. The use of living animals in research and testing became significant in the second half of the nineteenth century as part of the development of the emerging sciences of physiology and anatomy. The post-war expansion of the pharmaceutical and chemical industries saw an enormous increase in the use of animals in research. Today it is a multi-billion dollar industry, encompassing the pharmaceutical and chemical industries and university and government bodies. There is also a significant industry providing support services in relation to animal research, including animal breeding, food supply, cage manufacture, etc. The vast majority of animals used in research are subjected to some degree of pain or stress during experimental procedures to which they may be subjected, or as a result of the environment in which they are kept prior to or after those procedures. ${ }^{1-4}$

The physical component can be a cage, an isolator cabinet, an animal room, an area of a facility, the entire animal facility, or any combination thereof serving as primary, secondary and tertiary barriers. The management component involves operational procedures designed to prevent the introduction of undesirable infectious agents into the barrier. The most important factor for preventing entry of unwanted agents is a well-trained animal-care and research staff. The most common rodent barrier in use today is a cage-level barrier system referred to as the micro-isolation caging system or Individually
Ventilated Cages (IVC). This "system" combines the physical attributes of a micro-isolation cage and a biosafety cabinet (or similar HEPA filtered air cabinet/"cage-change station") with management procedures. Everything that the animals contact is to be sterilized: the cage, bedding, water and feed. The system is completed by opening the micro-isolation cage only inside the HEPA filtered air environment of a biosafety cabinet. A common component of all barriers is equipment for sterilizing cages and supplies that have direct contact with the animals. Typically this involves an autoclave, although other types of sterilization equipment are available. The human safety of medicines, agricultural chemicals and various other chemical products, such as shampoos, cleaners and so on, is assessed by testing the products on animals. ${ }^{3}$ Regulatory authorities responsible for the approval of the use of these substances in most countries require animal testing on the substances and it is likely that the requirements in western countries will be met by testing in India for substances intended to be marketed internationally. The tests involved are particularly repugnant, as they usually involve poisoning the animals concerned - death is often the endpoint measured. All these tests or studies must be carried out in GLP accreditated contract research organisations whose animal research facility is operating as per GLP norms. Animal Research Facility should be of adequate size and shape. In addition to animal experimental rooms with air handling and air conditioned along with clean and service corridors. There should be sufficient space available for washing cages and water bottles as well as racks. One or two rooms should be allocated for animal quarantine as well as storing feed and bedding materials. Animals should be monitored for their health therefore we need to have diagnostic laboratories to undertake these tests to prove your animals are free from any disease. Entry and exit to animal research facility should be monitored. Persons entering in to animal facility should ware proper protective devices and clothing. Entry should be through air shower is preferred to avoid any contamination.

Utilities (cages and water bottles) sent in to animal facility should be autoclaved and there should be sufficient space to store autoclaved materials in clean corridor side. We need to provide veterinary care to all experimental animals. Therefore this has to be done by attending veterinarian only. There should be one room dedicated for sick animals termed as containment room in addition to experimental rooms. Animals received in to Animal Research Facility, has to undergo quarantine of 5 to 7 days. There should be separate one or two quarantine rooms where animals can be quarantined and observed for clinical signs and found any diseased animals can be treated before it is used for any experimental purpose. One needs to decide the fate of the animals based on scientific judgment, attending veterinarian can decide the fate of the animal or the batch as a whole. All records of quarantine along with the veterinary certificate should be the part of study raw data. Animals after quarantine are moved in to experimental room wherein they will be subjected to acclimatization for 5 days, the thereafter randomisation can be done to segregate animals in to the experimental groups. Study can be acute, sub-acute, or chronic based on the study protocol/study plan.

\section{Animal research facility guidelines}

Entry into the animal research facility should be through air shower. The vestibule helps control air flow, preventing lab air from passing out into the corridor, and acts as an overall barrier against cross contamination 
a) Animal Research Facility for experimentation should be minimum of 6 to 12 rooms, individually ventilated with 10 to 15 air exchanges, 450lux illumination with 12 hour light and dark cycles and sound with 60 decibel, barrier maintained with clean and service corridors.

b) There should be separate quarantine facility with minimum of two rooms

c) There should be separate feed and bedding storage one room each

d) Washing area with big autoclave facility

e) Separate Necropsy area for postmortem

f) Separate laboratories for Biochemistry and Histology

g) Independent Histopathologist for slide observations

h) Separate Wet and Dry Archiving facility

i) Test and Reference item control Office

j) Refrigerated storage and/or freezer

k) Backup facility for electricity failure-DG set etc.

1) Reagent preparation and formulation room

m) Administrative work spaces for Test Facility Management/Study Directors/Document Control/Quality Assurance/IT-Data Entry and Statistics/Purchase and Finance etc.

These are general design guidelines for Animal Research Facility as per GLP norms. But this list does not account for the qualitative aspects of the facility. Laboratory design must address the quality of the work environment for all personnel. We need to focus on uses, its design solutions on people and strives to enhance the human factors in every facility. This is reinforced through a design producing an environment that enhances communication and promotes productivity within and between various department and sections of the laboratory. A variety of functional elements can drive the architectural and engineering design solution for a toxicology laboratory. Requirements such as: scientific process, and instrumentation are just a few of the variables that generate space, dimension, and adjacency requirements that affect the overall solution. There is not one universally correct solution for a toxicology lab; solutions will differ according to the needs of the industry. Flexibility is a key element in driving a laboratory's design and configuration. In recent years, one message in particular has been made clear to the scientific, architectural, and building industry professions: new laboratories must be designed with the flexibility to support adaptability and change. Adaptability in a laboratory is a function of how the buildings, rooms, and systems can grow and change with the needs of its occupants. For example, over the past several years scientific advancement in genotoxicity has forced laboratories to adapt to new designs in order to decrease the risk of contamination and ensure the accuracy of technical data. As science and technology changes, laboratories must be capable of embracing the future. Most of the Animal Research Facilities are different. Specific procedures and processes being carried out in the laboratory and specific situations will require different solutions. But all animal research laboratories possess similar characteristics as they are performing similar functions. The ideas and guidelines above provide general ideas for well designed animal research facility which is functioning as per Good Laboratory Practice.

\section{Discussion}

The purpose of biomedical research and testing is to understand the living body and what goes wrong in disease, and to develop safe and effective ways of preventing or treating those diseases. Animals are vital in all stages of this undertaking, not just in safety testing. The three Rs are the guiding principles in animal research. Research on laboratory animals is necessary for scientific research for generating new knowledge and information however when animals are used should be justified and approved by Institutional Animal Ethics Committee. Animal welfare is the responsibility of the scientist who is using them. We need to take care of the animals. Animal Research Facility which is designed as per Good Laboratory Practice and research is carried out as per study plan and standard operating procedures has worldwide acceptance under Mutually Acceptance of Data treaty.

\section{Conclusion}

Animals are used in research, when, there is a need to find out what happens in the whole, living body, which is far more complex than the sum of its parts. It is difficult to replace the use of living animals in research with alternative methods Adding to scientific knowledge through basic biological research helps us understand how living things work, and apply that understanding for the benefit of both humans and animals..Good Laboratory practice as conceived by US FDA/OECD provide right direction for using animals for research and data generated in GLP approved Research Laboratory can be used for international product registration.

\section{Acknowledgements}

None.

\section{Conflict of interest}

The authors declare that there is no conflict of interest.

\section{References}

1. Daniel Watch. Building type basics for research laboratories. 2nd ed. John Wiley \& Sons: New York; 2008.

2. Guide for the Care and Use of Laboratory Animals by the Institute of Laboratory Animal Resources. Commission on Life Sciences, National Research Council. Washington: National Academy Press; 1996.

3. ILAR Journal the quarterly, peer-reviewed publication of the Institute for Laboratory Animal Research.

4. Russell WMS, Burch RL. The Principles of Humane Experimental Technique. London: Methuen; 1959. 